\title{
EXIGENCIAS NUTRICIONALES DE PROTEÍNA BRUTA Y ENERGÍA METABOLIZABLE PARA POLLOS DE ENGORDE
}

\author{
NUTRITIONAL REQUIREMENTS OF CRUDE PROTEIN \\ AND METABOLIZABLE ENERGY FOR BROILERS
}

\author{
Diana M. Torres-Novoa \\ Médica Veterinario Zootecnista, Magister en Ciencia Animal, Estudiante de Doctorado en Ciencias Agrarias, \\ Escuela de Ciencias Agrícolas, Pecuarias y del Medio Ambiente (ECAPMA), Universidad Nacional \\ Abierta y a Distancia (UNAD), Acacias, Colombia. \\ milena.torres@unad.edu.co
}

\section{Resumen}

Esta revisión fue realizada con el objetivo de acopiar información de los recientes avances sobre las exigencias de proteína bruta y energía metabolizable para pollos de engorde. En la actualidad el concepto de exigencia de proteína bruta, está siendo sustituido por proteína ideal, especificando los niveles de aminoácidos.

Las exigencias nutricionales para pollos de engorde establecidas en los Estados Unidos, difieren a las exigencias nutricionales determinadas para pollos en condiciones tropicales. El ofrecimiento de raciones atendiendo las necesidades nutricionales para las diferentes etapas de la vida del pollo, permite alcanzar un buen desempeño zootécnico.

Palabras clave: alimentación, avicultura, ganancia de peso, nutrición, rendimiento en canal.

\begin{abstract}
This review was conducted with the objective to offer information from recent advances on the crude protein and metabolizable energy requirements for broilers. At present, the concept of crude protein requirement is being replaced by ideal protein, specifying aminoacid levels.
\end{abstract}

The nutritional requirement for broilers suggested by the United States differ from the estimates nutritional requirements for broilers under tropical conditions. Food supply for attend nutritional requirements in different phases of broilers, allows to achieve a good zootechnical performance.

Key words: feed, poultry, weight gain, nutrition, channel performance 


\section{Introducción}

Los desbalances nutricionales en la dieta afectan la calidad de los productos, los excesos de nutrientes aumentan la excreción de los mismos, lo cual contribuye al deterioro del ambiente, aumentando los costos de producción y reduciendo la rentabilidad de la actividad avícola (Bohórquez, 2014). Entretanto, la deficiencia de nutrientes no permite que los animales expresen todo su potencial productivo. Por lo anterior, el conocimiento de las exigencias nutricionales de las aves permitiría la utilización de materias primas que faciliten la reducción de los costos de producción sin afectar los resultados zootécnicos ni la calidad del producto. La proteína es un nutriente importante en la alimentación de aves, considerando que el propósito de la producción, es una eficiente conversión de proteína de la dieta en proteína muscular mediante la utilización óptima de energía contenida en los alimentos. Por otro lado, el exceso de proteína implica el catabolismo de los aminoácidos y la excreción como ácido úrico. De esta forma, la proteína en vez de ser utilizada para deposición en tejido es desviada para la excreción de nitrógeno (Gómez et al., 2011). Borges (2013) concluye que es posible reducir las pérdidas de nitrógeno en 30 a $40 \%$ con la reducción de la proteína bruta en la dieta. La energía resulta del metabolismo de las aves y es utilizada para el mantenimiento de los procesos vitales, como respiración, mantenimiento de la temperatura corporal y flujo sanguíneo, por su parte, el desempeño de las aves tiene relación directa con el nivel de energía en la dieta (Santos et al., 2012).

Para la elaboración de este documento fueron consultadas las principales bases científicas relacionadas con la nutrición de pollos, sistematizando la información de exigencias de proteína bruta (PB) y energía metabolizable (EM).
Para estimar las exigencias nutricionales se utilizan dos métodos, dosis-respuesta y factorial. El método dosis-respuesta, determina las exigencias con base en la respuesta del desempeño de los animales, alimentados con niveles crecientes del nutriente estudiado. El factorial, está basado en los principios de la determinación de la cantidad de nutriente que el animal necesita para el mantenimiento, crecimiento y producción. (Campos et al., 2008)

\section{Características del pollo de engorde}

Los pollos de engorde o "broilers" comenzaron a criarse hace unos sesenta años primero en los Estados Unidos y luego en Europa. El término "broiler" es aplicado a pollos y gallinas que han sido seleccionadas especialmente para rápido crecimiento, resistente a enfermedades y buena presentación física (Valdiviezo, 2012). En las aves se habla de líneas genéticas más que de razas, debido a que estas son híbridas y el nombre corresponde al de la empresa que las produce. Según Carranza et al. (2009) la obtención de las líneas broiler está basada en el cruzamiento de razas diferentes, utilizando normalmente las razas White Plymouth Rock o New Hampshire en las líneas madres y la raza White Cornish en la línea paterna. La línea paterna aporta las características de conformación de un animal de carne: tórax ancho y profundo, patas separadas, buen rendimiento de canal, alta velocidad de crecimiento, entre otras (Guilcapi, 2013).

\section{Métodos para determinar las exigencias nutricionales}

En el sector avícola los gastos en la alimentación constituyen el mayor valor de los costos totales de producción. Así, los nutricionistas han enfocado las investigaciones en las exigencias nutricionales de PB y EM para la elaboración de dietas balanceadas con mayor rentabilidad sin afectar negativamente los parámetros zootécnicos ni la calidad de la carne de las aves (Arabi., 2015; Oliveira et al., 2011). 
Según Campos et al. (2008) la exigencia de un nutriente puede ser definida como la cantidad a ser proporcionada en la dieta, para atender las necesidades de mantenimiento y producción, en condiciones ambientales acorde con la buena salud del animal. Para determinar las exigencias nutricionales en pollos de engorde, pueden ser utilizados dos métodos: dosis respuesta y factorial. El método dosisrespuesta, determina las exigencias con base en la respuesta del desempeño de los animales, alimentados con niveles crecientes del nutriente estudiado. El factorial, está basado en los principios de la determinación de la cantidad de nutriente que el animal necesita para el mantenimiento, crecimiento y producción (Campos, et al., 2008).

\section{Exigencia de proteína}

Las proteínas son biomoléculas formadas básicamente por carbono, hidrógeno, nitrógeno y oxígeno adicionadas en las dietas para el suministro de aminoácidos (Walsh, 2013). El exceso de este nutriente implica el catabolismo de los aminoácidos, funcionando como aporte de energía en las dietas. Esta función no es recomendable debido a su elevado costo como fuente energética. De esta manera, las dietas de pollos de engorde deben ofrecer un nivel proteico que minimice el uso de aminoácidos como fuente de energía (Bertechini, 2012).

El exceso de energía y proteína es convertido en grasa, debido a que las aves tienen gran facilidad de acumulo de grasa, por la baja capacidad de almacenar carbohidratos y proteínas y un tejido adiposo que acumula gran cantidad de grasa, así, el mecanismo genético que determina la síntesis de proteína es más complejo que el de síntesis de grasa; el exceso de nutrientes ingeridos, es utilizado en la síntesis de grasa (Brumano, 2009). Por su parte, reducir los niveles de proteína, disminuye el tejido magro y aumenta la grasa abdominal.
El alto contenido de grasa en la canal disminuye el valor comercial de los cortes y el rendimiento zootécnico, debido a las pérdidas de partes de tejido. Además, dificulta el adecuado tratamiento de los efluentes. En la práctica, cierta cantidad de grasa intramuscular es deseable para garantizar terneza y sabor a la carne de pollo (Brumano, 2009).

La edad afecta las exigencias nutricionales en las aves como lo evidencia Rostagno et al. (2011), los pollos de engorde necesitan $47,8 \mathrm{~g}$ de $\mathrm{PB} /$ kilogramo $(\mathrm{kg})$ de peso vivo en la primera semana de edad. Entre los 43 a 46 días, esta necesidad disminuye a $11,5 \mathrm{~g}$ de PB. En el caso del sexo, los machos presentan mayores exigencias nutricionales en comparación con las hembras en la misma edad.

Los niveles de 170 a $230 \mathrm{~g} / \mathrm{kg}$ de PB en dietas para pollos de engorde afectan negativamente el peso final, la ganancia diaria de peso (GDP), consumo de ración y conversión alimenticia (CA) (Rezaei et al., 2004; Figueiredo et al., 2012). A diferencia de los anteriores trabajos, el desempeño zootécnico, el peso de pechuga (Rezaei et al., 2004), ganancia de peso, conversión alimenticia, peso de la canal en pollos capones con diferentes niveles de proteína a base a quinua con $2860 \mathrm{Kcal}$ EM/Kg MS (Aguaguiña, 2016) y pernil no son afectados por este nutriente en condiciones de termoneutralidad (Oliveira et al., 2011). De igual manera, en dietas con aumento en la PB, se observó incrementos en la GDP y mejora en la CA (Gheorghe et al., 2013a; Plumstead et al., 2005).

Makinde \& Egbekun (2016) y Mahmoud et al. (2017) observaron con $22 \%$ de PB, mejor ganancia de peso, conversión alimenticia, digestibilidad de proteína bruta y rendimiento de la canal; en la etapa de crecimiento Guilcapi, (2013) observó con 20\% de PB, obtuvieron los mejores parámetros productivos 
en cuanto a ganancia de peso y conversión alimenticia y en la etapa de engorde con $18 \%$ de $\mathrm{PB}$, consiguieron los mejores promedios productivos en peso final, ganancia de peso y conversión alimenticia.

Asadi et al. (2017) observaron que la inclusión de gelatina tuvo efecto negativo sobre el rendimiento de crecimiento de la canal en pollos de engorde alimentados con dietas bajas en $\mathrm{PB}$, los autores argumentan, que el contenido de glutamina de dietas experimentales se redujo por los aumentos en los niveles de gelatina y las disminuciones fueron más evidentes en las dietas de bajo contenido proteico. La glutamina se considera como una fuente de nitrógeno para formar otros aminoácidos y su deficiencia da lugar a efectos más adversos sobre el rendimiento de los pollos de engorde con bajos niveles de PB (Berres et al., 2010).

Paraskeuas et al. (2016) concluyeron que al disminuir los niveles de energía y proteína afecta negativamente la conversión alimenticia, mientras que la suplementación de un aditivo alimenticio fitogénico a base de orégano, anís y aceites esenciales de cítricos compensan estos efectos.

Dietas bajas en proteína producen similares ganancias de peso y conversión alimenticia, mejorando estos parámetros con la suplementación de ácido orgánicos (Dehghani \& Jahanian, 2016), las aves ajustan el consumo de alimento a las necesidades de aminoácidos no esenciales en comparación con niveles estándar de proteína (Hernández, 2005).

Yang et al. (2016) observaron que la proteína y la energía se pueden disminuir en $10 \%$ de 8 a 14 días de edad del ave, Sarwar et al. (2015) con reducción en 0,50\% de PB y $50 \mathrm{kcal} / \mathrm{kg}$ EM se obtuvieron los mejores resultados productivos y de costos de producción y Basavanta et al. (2016) observaron que la PB puede disminuir en 0,75 y $1,5 \%$ con formulación de aminoácidos totales y aminoácidos ileal digeribles, respectivamente.

Liboni et al. (2013) encontraron rendimiento en canal, pechuga y muslo de 71,69; 25,12 y $22,06 \%$ respectivamente, para pollos de engorde de 42 días alimentados con $19,14 \%$ de PB y $0,98 \%$ de lisina en la dieta. Urdaneta \& Leeson (2004) encontraron en pollos de 0 a 21 días con $170 \mathrm{~g}$ de $\mathrm{PB} / \mathrm{kg}$ de alimento y $1,22 \%$ de lisina en la dieta, disminución del $12 \%$ en el rendimiento de pechuga.

Cevallos \& Intriago, (2014) recomendaron para máxima producción y mejor costo-beneficio para pollos Ross en fase inicial $20 \%$ de PB con suplementación de 0,45; 0,22 y $0,20 \%$ de lisina, metionina y treonina sintética, respectivamente. En fase de crecimiento $18 \%$ de PB con suplementación de 0,40 ; 0,20 y $0,25 \%$ de lisina, metionina y treonina sintética, respectivamente. En fase final $16 \%$ de PB con suplementación de 0,42; 0,20 y $0,28 \%$ de lisina, metionina y treonina sintética, respectivamente.

La reducción de PB en la dieta con la adición de aminoácidos sintéticos se obtienen resultados positivos en ganancia de peso y conversión alimenticia (Miranda et al., 2015; Vasconcellos et al., 2015), disminución en la excreción de nitrógeno (Awad et al., 2017; Gheorghe et al., 2013b) representando el 22,8\% por cada unidad porcentual de PB (Gomide, 2010). Sin embargo, Caetano et al. (2015) recomendaron no disminuir la PB en las primeras semanas de vida.

El contenido proteico de las dietas también fue estudiado en relación a la temperatura ambiente. Altas temperaturas reducen el consumo de alimento y la tasa de crecimiento. El 
ofrecimiento de dietas alta en PB para pollos sometidos a estrés por calor tiene un efecto perjudicial en la ganancia de peso, conversión alimenticia (Saki et al., 2016) y composición de canal. Dietas con niveles de PB bajos para pollos en estrés por calor aumenta la deposición de grasa en los cortes (Berres, 2010). Las recomendaciones de PB y EM para estas condiciones pueden ser de $23 \%$ y 3000 kcal según Arabi (2015).

Por las investigaciones expuestas en párrafos anteriores, las exigencias nutricionales para las aves pueden ser afectadas por la edad, función fisiológica, sexo, estado sanitario, condiciones de termoneutralidad, y tipo de alimentación.

\section{Exigencias de energía}

La energía no es un nutriente, es resultado del metabolismo de los componentes químicos de los alimentos, que es utilizada para funciones de metabolismo, crecimiento, producción, movimientos musculares, mantenimiento de la temperatura corporal, respiración, funcionamiento del aparato digestivo y síntesis de compuestos y procesos bioquímicos (Murarolli, 2007).

La energía total de un alimento nunca es completamente aprovechada por las aves, pues parte de esta energía se pierde con las heces y orina (Englert, 1998). Se considera dos maneras de medir el valor energético de las raciones y de las materias primas importantes en la formulación de raciones: Energía metabolizable y energía productiva. La energía metabolizable es la energía total del alimento menos la energía de las heces y orina y, la energía productiva es la energía de una ración que es realmente transformada en carne (Murarolli, 2007). García \& Quijia (2012) observaron que al disminuir $50 \mathrm{kcalEM} / \mathrm{kg}$ en la dieta no se afectó el peso corporal, consumo de alimento, conversión alimenticia ni ganancia de peso. De igual forma, Gitoee et al. (2015) concluyeron que los niveles de EM pueden reducirse con la adición de enzimas sin afectar el rendimiento de los pollos de engorde.

Varios autores observaron que nivel energético de $3000 \mathrm{kcal} / \mathrm{kg}$ en dietas para pollos de engorde no causa disminución del peso corporal y la conversión alimenticia es favorecida por el aumento de la EM de la ración, como consecuencia del aumento de la ganancia de peso y la disminución del consumo de ración (Infante et al., 2016; Perween et al., 2016; Ferreira et al., 2015).

Niveles de 3100 a $3300 \mathrm{kcalEM} / \mathrm{kg}$ no interfieren en el desempeño zootécnico y en el rendimiento de canal, pechuga, pernil, alas, pies y cabeza de pollos (Gris Barre Cou Plume) de 1 a 90 días de edad (Santos et al., 2012). Resultados semejantes fueron obtenidos por Santos et al. (2014) que no observaron efecto sobre el desempeño y el rendimiento de la canal en pollos de 28 días de edad de la línea Isa Label alimentados con $2750 \mathrm{kcal} / \mathrm{kg}$ EMAn (Energía metabolizable aparente corregida) y $19,80 \%$ de PB. El contenido de grasa intramuscular es inferior en aves que recibieron la ración con menor nivel energético (Da Silva et al. 2015).

Por otro lado, el suministro de 3350 kcalEM/ $\mathrm{kg}$ en la dieta de pollos de engorde proporcionó mejores resultados de desempeño y con 3200 kcalEM/kg mejoró la eficiencia en la utilización de energía para deposición de proteína y consecuentemente, mejor calidad de canal (Sakomura et al., 2004). En general las raciones energéticas y con ajustes en los nutrientes promueven mejor rendimiento en la etapa de finalización (Dutra, 2016).

La energía fue estudiada en relación a la temperatura ambiente, Oliveira (2015) concluyó 
que en condiciones de termoneutralidad o de estrés por calor, el aumento del nivel de EM en la ración, no influyó en el desempeño de los pollos de engorde con edad entre 21 y 42 días y proporcionó efectos distintos en las respuestas fisiológicas y en el rendimiento de la canal.

\section{Relación energía metabolizable: proteína bruta}

La relación energía metabolizable: proteína bruta es la cantidad de energía (kcal) para cada un gramo de proteína de la ración (Murarolli, 2007).

Cuando los niveles de proteína bruta son reducidos en dietas isoenergéticas, ocurre un aumento en la relación EM:PB, favoreciendo mayor deposición de grasa corporal, hecho que contrasta la actual preocupación del consumidor de reducir la cantidad de grasa en la dieta (Reginatto et al.2000).

La relación energía:proteína alta $(149,8 ; 174,4$ y 194,4, para las fases inicial, crecimiento y finalización, respectivamente) en machos en los periodos de 1 a 35 días y de 1 a 48 días presentó mejor conversión alimenticia y mayor porcentaje de grasa abdominal (MuraroIli, 2007). Raciones con relación EM:PB baja $(130,0 ; 150,0$ y 166,7 para las fases inicial, crecimiento y finalización, respectivamente) posibilita menor tasa de grasa abdominal, resultando en mayor cantidad de carne magra en la canal, lo que es de gran ventaja para el mercado consumidor y para el productor en relación al costo-beneficio (Murarolli, 2007). Según Mendonça et al. (2008) la relación calórica: proteica recomendada para machos Isa Label en la fase inicial es de 128 y Mushtaq et al., (2015) deducen que el mejor rendimiento zootécnico puede obtenerse con una relación de 11,4 EM/PB y $1,05 \%$ de lisina manteniendo todos los otros AA constantes bajo condiciones subtropicales de verano.

\section{Consideraciones finales}

Las exigencias de proteína se han reportado en un rango de 18 a $23 \%$, según la etapa de desarrollo biológico. Los de energía metabolizable entre 2950 a $3350 \mathrm{kcalEM} / \mathrm{kg}$ para obtener los mejores resultados de desempeño.

Las exigencias nutricionales descritas han sido determinadas en otros países, haciendo necesario que en Colombia se evalúen proyectos de investigación con el objetivo de estimar las exigencias nutricionales para pollos de engorde en zonas tropicales.

En la formulación de dietas prácticas se debe considerar un margen de seguridad en la atención de las exigencias nutricionales bien sea por ingredientes diferentes, línea genética, temperatura ambiente, consumo del alimento, estado sanitario, edad y sexo del ave.

\section{Literatura citada}

Aguaguiña, T. D. A. (2016). Comportamiento productivo de pollos capones pio pio con dietas isoeléctricas y diferentes niveles de proteína en base a quinua. (Tesis de pregrado). Escuela Superior Politécnica de Chimborazo. Riobamba, Ecuador.

Arabi, S. A. M. (2015). The Effects Of Different Protein And Energy Levels On Broilers Performance Under Hot Climatic Conditions. International Journal of Innovative Agriculture \& Biology Research, 3(1), 19-28.

Asadi Z. K. K., Shahir, M. \& Baradaran, N. (2017). Effect of gelatin supplementation on growth performance and blood metabolites of broiler chickens fed diets varying in crude protein. Livestock Science, 201, 5-12.

Awad, E. A., Zulkifli, I., Soleimani, A. F. \& Aljuobori, A. (2017). Effects of feeding male and female broiler chickens on low-protein diets fortified with different dietary glycine levels under the hot and humid tropical climate. Italian Journal Of Animal Science, 16(3), 453-461.

Basavanta, K. C., Gloridoss, R. G., Singh, K. C., Prabhu, T. M. \& Suresh, B. N. (2016). Performance of Broiler Chickens Fed Low Protein, Limiting 
Amino Acid Supplemented Diets Formulated Either on Total or Standardized Ileal Digestible Amino Acid Basis. Asian-Australasian Journal of Animal Sciences, 29(11), 1616-1624.

Berres, J. (2010). Estratégias para redução protéica de dietas para frangos de corte. (Teses de Doutorado). Universidade Federal do Rio Grande do Sul. Porto Alegre-RS, Brasil.

Berres, J., Vieira, S. L., Dozier, W. A., Cortês, M. E. M., Barros, R., Nogueira, E. T. \& Kutschenko, M. (2010). Broiler responses to reduced-protein diets supplemented with valine, isoleucine, glycine, and glutamic acid. Journal Applied Poultry Research, 19, 68-79.

Bertechini, A. G. (2012). Niveles de proteína y aminoácidos en avicultura. Universidade Federal de Lavras, MG/Brasil. Recuperado de: http://amevea-ecuador.org/web_antigua/memorias2012/ memorias/PROTEINA_AMINOACIDOS_EN_AVICULTURA_DR_BERTECHINI.pdf

Bohórquez, A. V. D. (2014). Perspectiva de la producción avícola en Colombia. (Especialización en alta gerencia). Universidad Militar Nueva Granada. Bogotá, Colombia.

Borges, F. C. (2013). Redução da proteína bruta da ração de frangos de corte tipo caipira. (Dissertação). Universidade Federal dos Vales do Jequitinhonha e Mucuri. Diamantina-MG, Brasil.

Brumano, G. (2009). Níveis de lisina e de metionina + cistina e proteína bruta para melhor qualidade de ovo e de carcaça de aves e suínos. Nutritime, 6(3), 898-917.

Caetano, V. C., Faria, D. E., Caniatto, A. M., Faria Filho, D. E. \& Nakagi, V. S. (2015). Performance and carcass yield of broilers from 1 to 46 days fed diets containing different levels of valine and reduced protein content. Arquivo Brasileiro de Medicina Veterinaria e Zootecnia, 67(6), 1721-1728.

Campos, A., Salguero, S., Albino, L. \& Rostagno, H. (Novembro de 2008). Aminoácidos en la nutrición de pollos de engorde: proteína ideal. III CLANA- Congresso do Colégio Latino-Americano de Nutrição Animal. Congreso llevado a cabo en Cancún, México.

Carranza, C. K. M. \& Díaz, O. M. (2009). Efecto de la caponizacion en pollos de engorde (línea hubbard) y de postura (línea Hyline Brown) en el peso y las características organolépticas de la canal. (Tesis de pregrado). Universidad del Salvador. San Salvador.
Cevallos, V. L. E, \& Intriago, Z. C. R. (2014). Utilización de aminoácidos sintéticos y diferentes niveles de proteína en la optimización de parámetros de producción de pollos Ross. (Tesis de Pregrado). Universidad Técnica de Manabí. Portoviejo. Manabí, Ecuador.

Da Silva Ferreira, G., Pinto, M. F., Neto, M. G., Ponsano, E. G., Gonçalves, C. A., Bossolani, I. C. \& Pereira, A. G. (2015). Ajuste preciso do nível de energia na dieta de frangos de corte para controle do desempenho e da composição lipídica da carne. Ciência Rural, 45(1), 104-110.

Dehghani, T. N. \& Jahanian, R. (2016). Effect of supplemental organic acids on performance, carcass characteristics, and serum biochemical metabolites in broilers fed diets containing different crude protein levels. Animal Feed Science and Technology, 211, 109-116.

Dutra, L. J. (2016). Níveis de energia e nutrientes para frangos de corte: desempenho, rendimento de carcaça e expressão gênica. (Dissertação) Universidade Federal de Sergipe, Brasil.

Englert, S. I. (1998). Nutrição correta-O segredo do sucesso. Avicultura, tudo sobre raças. Manejo e nutrição. Porto Alegre, Brasil. 7. Ed.: Editora Agropecuária.

Ferreira, S. G., Pinto, M. F., Neto, G. M., Ponsano, G. E. H., Gonçalves, C.A., Bossolani, C. I. L. \& Perreia, G. A. (2015). Ajuste preciso do nível de energia na dieta de frangos de corte para controle do desempenho e da composição lipídica da carne. Ciência Rural, 45(1), 104-110.

Figueiredo, V. C. H., Oliveira, F. D., Silva, S. C. G., Zacché, B. V. T., Almeida, S. M., Costa, M. A. L., Sabino, F. I. \& Medeios, V. F. (2012). Efeitos da redução da proteína dietética sobre o desempenho e as características de carcaça de frangos de corte de 1 a 21 dias de idade. Revista Brasileira de Zootecnia, 41(3), 662-667.

García, B. R. D. \& Quijia, P. J. O. (2012). Parámetros productivos del pollo de engorde sometido a dos niveles de energia entre los 22 a 35 días de edad. Escuela Agrícola Panamericana. (Tesis Pregrado). Zamorano, Honduras.

Gheorghe, A., Dragotoiu, D., Ciurescu, G., Lefter, N. \& Habeanu, M. (2013a). Effects of Dietary Protein Level on Protein Deposition in Broilers: 1. Body Composition, Plasma Metabolic Profile and Litter Composition. Bulletin UASVM Animal Science and Biotechnologies, 70(2), 266-273. 
Gheorghe, A., Dragotoiu, D., Ciurescu, G., Lefter N. \& Habeanu, M. (2013b). Effects of Dietary Protein Level on Protein Deposition in Broilers: 2. Body Composition, Plasma Metabolic Profile and Litter Composition. Bulletin UASVM Animal Science and Biotechnologies, 70(2), 274-283.

Gitoee, A., Janmohammadi, H., Taghizadeh, A. \& Rafat, S. A. (2015). Effects of a multi-enzyme on performance and carcass characteristics of broiler chickens fed corn-soybean meal basal diets with different metabolizable energy levels. Journal of Applied Animal Research, 43(3), 295-302.

Gómez, R. S., López, C. C., Cortés, C. A. \& Ávila, G. E. (2011). Evaluación de tres programas de alimentación para pollos de engorda con base en dietas sorgo-soya con distintos porcentajes de proteína. Veterinaria México, 42(4), 299-309.

Gomide, E. M. (2010). Redução dos níveis de proteína bruta, calcio e fosforo em rações com fitase e aminoácidos para frangos de corte. (Tese de Doutorado). Universidade de Lavras. Minas Gerais, Brasil.

Guilcapi, P. R. S. (2013). Utilización de aminoácidos sintéticos con reducción de proteína bruta en la alimentación de pollos parrilleros. Escuela Superior Politécnica de Chimborazo. (Tesis de Grado). Riobamba, Ecuador.

Hernández, S. C. (2005). Efecto de dietas bajas en proteína y niveles de lisina sobre el desarrollo dinámico del músculo de pechuga en pollos de engorda. (Tesis de Doctorado). Universidad Autónoma de Chihuahua. Chihuahua, México.

Infante, R. F., Salinas, C. J., Montaño, G. M. F., Manríquez, N. O. M., González, V. V. M., Guevara, F. O. F. \& Ramírez, L. J. A. (2016). Effect of diets with different energy concentrations on growth performance, carcass characteristics and meat chemical composition of broiler chickens in dry tropics. SpringerPlus, 5(1), 1937.

Liboni, B. S., Yoshida, S. H., Pacheco, F. P., Souza, L. F. A., Astolphi, J. L. \& Astolphi M. Z. (2013). Diferentes programas de luz na criação de frangos de corte. Revista Científica Electrônica de Medicina Veterinária, 20, 1-19.

Mahmoud, K., Obeidat, B., Al-Sadi, M. \& Hatahet, S. (2017). Effect of Bacillus subtilis supplementation and dietary crude protein level on growth performance and intestinal morphological changes of meat type chicken. Livestock Science, 195, 99-104.
Makinde, O. A. \& Egbekun, C. P. (2016). Determination of optimum dietary energy and protein levels for confined early-stage Fulani Ecotype chickens. Livestock Research for Rural Development, 28(9), 164.

Mendonça, O. M., Sakomura, N. K., Santos, F. R., Freitas, R. E., Fernandes, K. B. J. \& Barbosa, A. N. A. (2008). Níveis de energia metabolizable para machos de corte de crescimento lento criados em semiconfinamento. Revista Brasileira de Zootecnia, 37(8), 1433-1440.

Miranda, D. J. A., Vieira, S. L., Favero, A., Angel, C. R., Stefanello, C. \& Nogueira, E. T. (2015). Performance and meat production of broiler chickens fed diets formulated at different crude protein levels supplemented or not with L-valine and L-isoleucine. Animal Feed Science and Technology, 206, 39-47.

Murarolli, R. A. (2007). Efeitos de diferentes relações dietéticas de energia metabolizable: proteína bruta e do peso inicial de pintos sobre o desempenho e o rendimento de carcaça em frangos de corte: I machos; II fêmeas. (Dissertação). Universidade de São Paulo. Pirassununga. Brasil.

Mushtaq, M. M. H., Ahmad, G. \& Parvin, R. (2015). Influence of dietary graded levels of digestible lysine and lowering energy to protein ratio on the growth performance of broilers under subtropical summer conditions. Revista Brasileira de Ciencia Avicola, 17 (3), 381-386.

Oliveira, P. W., Oliveira, M. R. F., Donzele, L. J., Albino, T. L. F., Martins, S. M. \& Assis, A. P. (2011). Redução do nível de proteína bruta em rações para frangos de corte em ambiente de termoneutralidade. Revista Brasileira de Zootecnia, 40(8), 1725-1731.

Oliveira, K. P. (2015). Influência de diferentes temperaturas e níveis de energia metabolizável no desempenho de frangos de corte na fase final de criação. (Dissertação). Universidade Federal de Viçosa. Viçosa, MG, Brasil.

Paraskeuas, V., Fegeros, K., Palamidi, I., Mountzouris, K. C. \& Theodoropoulos, G. (2016). Phytogenic Administration and Reduction of Dietary Energy and Protein Levels Affects Growth Performance, Nutrient Digestibility and Antioxidant Status of Broilers. Journal of Poultry Science, 53(4), 264-273.

Perween, S., Kumar, K., Chandramoni, Kumar, S., Singh, P. K., Kumar, M. \& Dey, A. (2016). Effect 
of feeding different dietary levels of energy and protein on growth performance and immune status of Vanaraja chicken in the tropic. Veterinary World, 9(8), 893-899.

Plumstead, P., Paton, N., Romero, S. H., Leksrisompong, N. \& Brake, J. (January 2005). Effect of dietary crude protein and amino acid balance on the early body weight gain response of broilers to dietary lysine. International Poultry Science Forum. Georgia World Congress Center, Atlanta, Georgia.

Reginatto, M. F., Ribeiro, A. M., Penz J. R, A. M., Kessler, A. M. \& Krabbe, E. L. (2000). Suplementação de Treonina em Dietas de Frangos de Corte, Variando a Energia e as Relações Energia:Proteína. Revista Brasileira de Ciencias Avícola, 2(3), 239-247.

Rezaei, M., Moghaddam, H. N., Reza, J. P. \& Kermanshahi, H. (2004). The effects of dietary protein and lysine levels on broiler performance, carcass characteristics and $\mathrm{N}$ excretion. International Journal Poultry Science, 3(2), 148-152.

Rostagno, H. S., Albino, L. F. T., Donzele, J. L., Gomes, P. C., Oliveira, R. F., Lopes, D. C., Ferreira, A. S., Barreto, S. L. \& Euclides, R. F. (2011). Tabelas brasileiras para aves e suínos, Composição de alimentos e exigências nutricionais. 3a Ed. Universidade Federal de Viçosa, Brasil.

Saki, A. A., Maleckey, M., Johari, R., Goudarzi, S. M. \& Abdolmaleki, M. (2016). The effects of protein, amino acid, and dietary electrolyte balance on broiler chicken performance and blood parameters under heat stress. Acta Scientiarum - Animal Sciences, 38(3), 285-292.

Sakomura, N. K., Longo, F. A., Boa-Viagem, R. C., Watanabe, K., Pelícia, K. \& Rodrigues, F. E. (2004). Efeito do nível de energia metabolizável da dieta no desempenho e metabolismo energético de frangos de corte. Revista Brasileira de Zootecnia, 33(6) (supl. 1), 1758-1767.
Santos, F. R., Stringhini, J. H., Minafra, C. S., Almeida, R. R., Oliveira, P. R., Duarte, E. F; Silva, R. B. \& Café, M. B. (2014). Formulação de ração para frangos de corte de crescimento lento utilizando valores de energia metabolizável dos ingredientes determinada com linhagens de crescimento lento e rápido. Arquivo Brasileiro de Medicina Veterinária y Zootecnia, 66(6), 1839-1846.

Santos, M. S. V., Vieira, S. S., Tavares, F. B., Andrade, P., Manno, M. C., Costa, H. S. \& Moreira, A. (2012). Desempenho, carcaça e cortes de frangos caipira francês barre (Gris Barre Cou Plume). Archivos de Zootecnia, 61(234), 287-295.

Sarwar, G. A. S., Khan, S. H., Anjum, M. A. \& Nadeem, M. A. (2015). Effect of different dietary protein and energy levels on the growth performance, meat and body fat composition in broiler chicks. Pakistan Journal of Agricultural Sciences, 52(4), 1125-1129.

Urdaneta, R. M. \& Leeson, S. (2004). Muscle (Pectorais Major) protein turnover in Young broiler chickens fed graded levels of lysine and crude protein. Poultry Science, 83, 1897-1903.

Valdiviezo, H. M. F. (2012). Determinación y comparación de parámetros productivos en pollos broiler de las líneas Cobb 500 y Ross 308, con y sin restricción alimenticia. (Tesis de grado). Escuela Superior Politécnica del Chimborazo. Riobamba. Ecuador.

Vasconcellos, C. F., Fontes, D. O., Lara, L. C., Silva, M. A., Vidal, T. B., Fernandes, I. S. \& Rocha, J. S. R. (2015). Evaluation of glycine plus serine levels in low crude protein diets for broiler chickens. Arquivo Brasileiro De Medicina Veterinaria e Zootecnia, 67(2), 499-505.

Walsh, C. P. (2013). Protein. Salem Press Encyclopedia Of Science.

Yang, H. M., Wang, W., Wang, Z. Y., Yang, Z., Huang, K. H., Lu, H., Wan, Y. \& Hou, B. H. (2016). Journal of Integrative Agriculture, 15(8), 1825-1832. 
